\title{
Inhibition Suppresses Transmission of Tonic Vibrissa-Evoked Activity in the Rat Ventrobasal Thalamus
}

\author{
Jed A. Hartings and Daniel J. Simons \\ Department of Neurobiology, University of Pittsburgh, Pittsburgh, Pennsylvania 15261
}

\begin{abstract}
Previous studies have demonstrated that tonic responses of trigeminal ganglion neurons to maintained whisker deflections are transformed to mainly phasic responses in thalamocortical neurons. The high tonic responsiveness of thalamic reticular neurons suggests that thalamic inhibition may contribute to this suppression of tonic activity. To test this hypothesis we recorded responses of thalamocortical neurons in the ventroposterior medial (VPm) nucleus to 200 and 400 msec sustained whisker deflections during simultaneous microiontophoresis of the GABA receptor antagonists bicuculline and phaclofen. Under control conditions, VPm units responded to deflection plateaus with mean activities of only 18 spikes/sec, compared with 16 spikes/sec spontaneous firing. A minority of cells $(5 / 19)$ had significantly greater plateau than spontaneous activity, and these cells were classified as tonic; the other 14/19 were considered phasic. Under GABA receptor antagonism, however,
\end{abstract}

A principal submodality classification of low-threshold mechanoreceptors and central neurons postsynaptic to them is based on their slowly versus rapidly adapting responses to sustained stimulation applied to their receptive fields. In monkeys, populations of slowly and rapidly adapting neurons remain segregated through synaptic relays and are aggregated in distinct functional columns in primary somatosensory cortex (Powell and Mountcastle, 1959; Dykes et al., 1981). In the rat trigeminal system, most cortical neurons respond only phasically to whisker deflection (Simons and Carvell, 1989), raising the issue of whether slowly adapting responses are masked by central inhibition.

Zucker and Welker (1969) found that $60 \%$ of primary afferent neurons innervating the whiskers were slowly adapting (tonically responsive), whereas thalamocortical neurons in the ventroposterior medial (VPm) nucleus have been described as primarily rapidly adapting, or phasic (Waite, 1973; Sugitani, 1979; Ito, 1988; Lee et al., 1994a,b). Similar results were obtained by Simons and colleagues, who characterized the responses of both primary afferent and V Pm populations using the same stimulation protocols and quantitative analyses. In the trigeminal ganglion (NV), $75 \%$ of neurons were found to respond to whisker deflections in a slowly adapting fashion and discharged at a mean rate of 70 spikes/sec above spontaneous levels during maintained deflections (Lichtenstein et al., 1990). In the thalamus, by contrast, a smaller proportion (45\%) of VPm cells were tonically responsive, and these cells maintained discharges at only 23 spikes/sec above spontaneous levels (Simons and Carvell, 1989; Hartings et al., 2000).

The transformation of response adaptation qualities between

Received May 31, 2000; revised July 11, 2000; accepted July 20, 2000.

This work was supported by National Institute of Neurological Disorders and Stroke Grant NS-19950 and National Institute of Mental Health Grant MH-61372.

Correspondence should be addressed to Dr. Daniel J. Simons, Department of Neurobiology, E1440 Biomedical Science Tower, University of Pittsburgh School of Medicine, Pittsburgh, PA 15261. E-mail: cortex+@pitt.edu.

Copyright (C) 2000 Society for Neuroscience $0270-6474 / 00 / 200001-05 \$ 15.00 / 0$ mean plateau activity increased to 53 spikes/sec compared with 30 spikes/sec spontaneous activity, and 7 of the 14 phasic units became tonically responsive. Increases in plateau activity were significantly greater, by both absolute and relative measures, than increases in spontaneous activity. Transient responses to stimulus onsets and offsets also increased in magnitude 4.0- and 2.9-fold, attributable mainly to their increased duration. These data indicate that VPm neurons receive tonic excitatory inputs that under normal conditions are masked by inhibition. Suppression of tonic activity in VPm by inhibitory thalamic reticular neurons may reduce tonic inhibition in cortical layer IV circuits, preserving their responsiveness to transient signals.

Key words: whisker; thalamocortical; reticular nucleus; inhibition; microiontophoresis; bicuculline primary afferent and thalamic neurons might be attributable to either a preferential relay of rapidly adapting inputs from the brainstem to VPm or to central inhibitory processes. The former possibility was judged unlikely by Lichtenstein et al. (1990) because many thalamic neurons have opponent directional preferences for ON and OFF responses, which is a characteristic mainly associated with slowly adapting NV neurons. In vibrissa neurons of cat VPm, simultaneous recordings of rapidly adapting units and their dendritic prepotentials suggested that VPm neurons in fact do receive slowly adapting inputs that are not expressed in their spike output (Gottschaldt et al., 1983). Consistent with this, disinhibition of rat VPm neurons prolongs the duration of their stimulus-evoked responses (Lee et al., 1994a,b). On the other hand, Vahle-Hinz and Hicks (1996) reported that tonic responses could not be evoked in VPm neurons with application of GABA receptor antagonists bicuculline and saclofen, suggesting an absence of slowly adapting trigeminothalamic input.

Recent recordings in our laboratory of thalamic reticular $(\mathrm{Rt})$ neurons, which are the only source of inhibition onto V Pm cells (Spacek and Lieberman, 1974; Harris and Hendrickson, 1987; Pinault et al., 1995), revealed high sustained discharge rates during maintained whisker deflections that might suppress ex-

This article is published in The Journal of Neuroscience, Rapid Communications Section, which publishes brief, peerreviewed papers online, not in print. Rapid Communications are posted online approximately one month earlier than they would appear if printed. They are listed in the Table of Contents of the next open issue of JNeurosci. Cite this article as: JNeurosci, 2000, 20:RC100 (1-5). The publication date is the date of posting online at www.jneurosci.org.

http://www.jneurosci.org/cgi/content/full/4576 
pression of slowly adapting inputs to VPm neurons (Hartings et al., 2000). We therefore reexamined the effects of inhibition on the tonic/phasic nature of VPm responses by microiontophoretically applying GABA receptor antagonists onto recorded VPm neurons.

\section{MATERIALS AND METHODS}

Surgical procedures. Adult Sprague Dawley rats weighing 250-300 gm were prepared for electrophysiological study using methods described previously in detail (Simons and Carvell, 1989). Halothane anesthesia was used during surgical procedures. A steel post was fixed to the skull with dental acrylic to hold the animal's head, and a craniectomy was made overlying the right V Pm nucleus (2.0-4.5 posterior, 1.5-4.0 lateral to bregma). After surgery, halothane was discontinued, the animal was immobilized by pancuronium bromide, artificially respired through a tracheal cannula, warmed by a servo-controlled heating blanket, and maintained in a lightly narcotized state by a steady infusion of fentanyl (Sublimaze, Jansen Pharmaceuticals) $\left(\sim 10 \mu \mathrm{g} \cdot \mathrm{kg}^{-1} \cdot \mathrm{h}^{-1}\right)$. The animal's condition was assessed by continuously monitoring electroencephalogram, femoral arterial blood pressure, tracheal airway pressure, and pupillary reflexes. At the end of recording sessions, animals were deeply anesthetized with sodium pentobarbital (Nembutal) and perfused transcardially. Brains were sectioned in the coronal plane and stained with thionin to confirm the location of electrode tracks through VPm.

Electrophysiological recordings and microiontophoresis. Initial localization and mapping of V Pm were performed with high-resistance stainless steel microelectrodes (Frederick Haer, Brunswick, ME). Three-barrel glass micropipettes with a carbon fiber recording channel were then used for simultaneous single-unit recordings and microiontophoresis. As described fully in Kyriazi et al. (1996), a carbon fiber $(\sim 8 \mu \mathrm{m}$ diameter $)$ was inserted into a single barrel of an assembly of three glass capillary tubes (A-M Systems, Inc., Everett, WA), and the assembly was pulled to a total tip diameter of $\sim 12 \mu \mathrm{m}$. The carbon fiber was then electrochemically etched to a conical tip and positioned within $20 \mu \mathrm{m}$ of the glass tip. The remaining two barrels were filled with $5 \mathrm{~mm}$ bicuculline methiodide (BMI) and $10 \mathrm{~mm}$ phaclofen in $0.9 \% \mathrm{NaCl}, \mathrm{pH} \mathrm{3.0.} \mathrm{Iontophoretic}$ electrodes had resistances of $30-60 \mathrm{M} \Omega$, and retaining currents of -20 nA were applied.

To antagonize GABA receptors, both BMI and phaclofen were always applied simultaneously, and the same current levels were used to eject each drug. For each recorded neuron, ejection currents were applied at progressively increased levels until a unit's spontaneous activity was substantially elevated (typically twofold) or responses to whisker deflection onsets and offsets were noticeably prolonged. The latter constituted the most common and reproducible effect of BMI/phaclofen application. Typically, currents of phaclofen and BMI were first applied at $25 \mathrm{nA}$ and then tested for an effect by 80 whisker deflection trials. Peristimulus time histograms (PSTHs) and spike counts over specified time periods were displayed online to examine effects on both spontaneous and stimulusevoked activities. If no changes were observed, currents were incremented in $25 \mathrm{nA}$ steps until an effect was achieved or $150 \mathrm{nA}$ was reached.

Whisker stimulation. Hand-held probes were used to identify the whisker evoking the strongest response from an isolated unit, i.e., the principal whisker. A piezoelectric mechanical stimulator was then attached to this whisker $10 \mathrm{~mm}$ from the skin surface (Simons, 1983). The stimulation protocol consisted of a ramp-and-hold deflection of the whisker from its resting position (Simons and Carvell, 1989). Deflections were $1 \mathrm{~mm}$ in amplitude $\left(\sim 5.7^{\circ}\right)$, with onset and offset velocities of $125 \mathrm{~mm} / \mathrm{sec}$ and a plateau duration of $200 \mathrm{msec}$. For some cells, $400 \mathrm{msec}$ plateau durations were also used. Deflections were applied in eight randomly interleaved directions spanning $360^{\circ}$ in $45^{\circ}$ increments and were repeated for 10 trials, for a total of 80 stimuli. Three seconds were allowed between each whisker deflection trial.

Data analysis. A time/amplitude window discriminator (BAK Electronics) and digital storage oscilloscope were used to isolate single units. Sequential spike event times were recorded with $100 \mu \mathrm{sec}$ resolution on a DEC LSI 11/73. Responses to the onset and offset of vibrissa deflections in each direction were computed as the mean number of spikes recorded for all 10 trials during $20 \mathrm{msec}$ epochs after the onset and offset of vibrissa deflections (ON and OFF responses, respectively). This time window was chosen based on the duration of responses under control conditions. Longer response windows were used to compute data in Table 1, as noted. Responses to the stimulus plateau, when the vibrissa was maintained in a deflected state, were measured during a $100 \mathrm{msec}$ period beginning $75 \mathrm{msec}$ after stimulus onset. Spontaneous activity was measured during a $100 \mathrm{msec}$ period preceding the deflection. Maximally effective (preferred) directions for ON and plateau responses were defined as those that evoked the most activity during the corresponding time epochs.

The "tonic" or "phasic" nature of each unit's response was assessed using a procedure used in previous studies (Simons and Carvell, 1989; Lichtenstein et al., 1990) and is similar to those used by others (Alloway et al., 1994; Lee et al., 1994b). One-tailed $t$ tests were used to compare spontaneous firing rates with those evoked during the stimulus plateau. A cell was classified as tonically responding if the plateau response at the maximally effective direction was significantly greater than spontaneous firing $(p<0.05)$; otherwise, the cell was classified as phasic. Two measures were used to assess a cell's directional tuning. Tuning ratios were computed as the response magnitude evoked at the maximally effective direction divided by the response averaged over all eight directions (Kyriazi et al., 1996); a higher value denotes greater selectivity. In a second analysis, each unit was classified into one of eight tuning categories based on the number of deflection angles $(0-7)$ that evoked responses statistically smaller than those of the maximally effective direction $(p<0.05$, one-tail) (Simons and Carvell, 1989); category 7 represents the most selective responses. Finally, the directional consistency of a unit's response was measured by computing the correlation between ON and OFF response magnitudes over the eight directions. Directionally consistent cells respond to either stimulus onsets or offsets, depending on direction, and therefore have negative correlation values. Data analyses and statistical tests were performed on a PC using Microsoft Excel/Visual Basic and the statistics package from SPSS, Inc.

\section{RESULTS}

We recorded the activity of 19 VPm neurons in three animals under control conditions and during simultaneous microiontophoresis of the $\mathrm{GABA}_{\mathrm{A}}$ receptor antagonist, BMI, and the $\mathrm{GABA}_{\mathrm{B}}$ receptor antagonist, phaclofen. For each cell, trapezoidal ramp-and-hold deflections were applied to the principal whisker, and iontophoretic currents were increased progressively until either the spontaneous or whisker-evoked activity were substantially elevated; $50-100 \mathrm{nA}$ was sufficient in most cases, as reported in other studies of VPm neurons (Hicks et al., 1986; Salt, 1989; Lee et al., 1994b). In a separate control experiment, six cells were studied using ejection currents applied via an inert pipette barrel $(0.9 \% \mathrm{NaCl}, \mathrm{pH} 3.0)$. These currents alone had no effect on either spontaneous or stimulus-evoked firing, although the same cells' activities were increased by BMI in a fashion similar to that reported below.

Figure 1 illustrates the effects of disinhibition on four neurons. Under control conditions, ON and OFF responses are brief, and sustained discharges during stimulus plateaus (i.e., maintained deflections) are minimal or absent. By contrast, under GABA receptor antagonism, $\mathrm{ON}$ and OFF responses are longer in duration, and many cells discharge continuously during the stimulus plateau. We classified cells as tonic if their activity during stimulus plateaus was significantly greater than spontaneous levels; all others were considered to be phasic. Under control conditions 14 of 19 cells were phasic. Under GABA antagonism, however, 7 of the 14 phasic neurons became tonic (Fig. $1 A, B$ ), and the mean plateau activity for these cells increased from 18 to 53 spikes/sec. For three other cells classified as phasic under control conditions, plateau activity increased significantly with drug application but was not greater than the elevated levels of spontaneous activity (Fig. 1C,D). Of the five cells classified as tonic under control conditions, mean plateau activity increased from 24 to 88 spikes/sec.

The units in Figure $1, A, C$, and $D$, exhibit directional consistency under control conditions; that is, they respond to the onset of deflections in one direction and to the offset of deflections in the opposite direction. Among primary afferent neurons, directional consistency is more often associated with slowly adapting than rapidly adapting responses (Lichtenstein et al., 1990). We found that directional consistency in VPm neurons was positively correlated with the extent of increase in plateau activity (all directions: $R^{2}=0.44$, linear regression $p=0.063$; preferred direction: $R^{2}=0.48$, linear regression $p=0.038$ ). This is consistent with the notion that increased plateau activity during $\mathrm{BMI} /$ phaclofen application is caused by the unmasking of excitatory inputs, because, on the basis of the responses of primary afferent neurons, directionally consistent VPm neurons are more likely to receive slowly adapting inputs.

Mean effects of GABA receptor antagonism are shown in the population PSTHs of Figure 2 and are quantified in Table 1. 
A
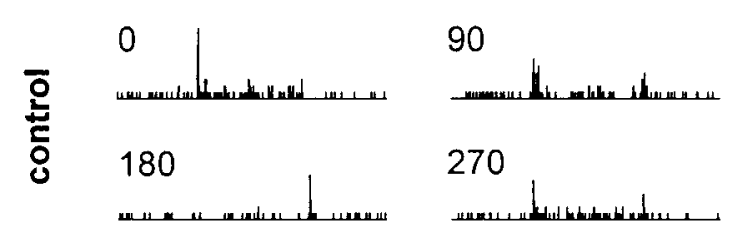

B
$D$
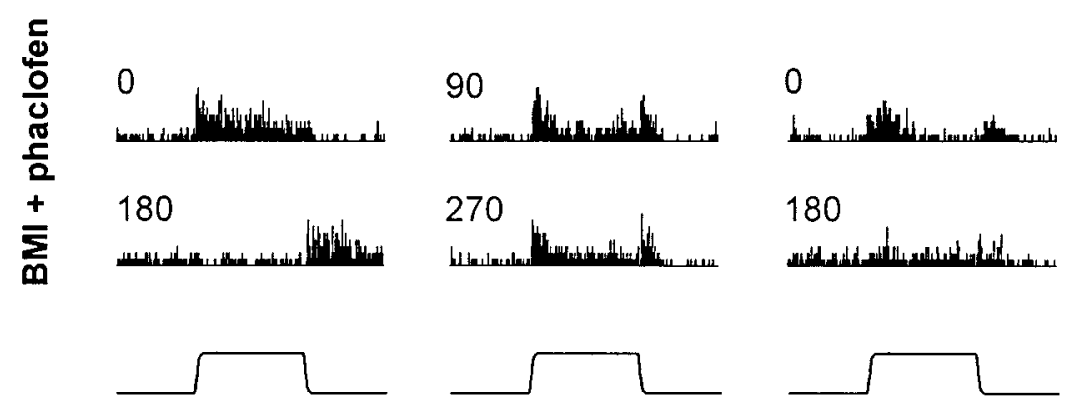

0

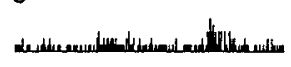

180

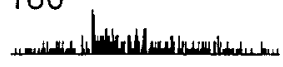

0

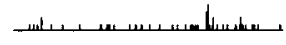

180

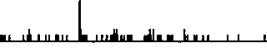

Figure 1. Disinhibition of single unit responses. $A-D$ each show responses of single VPm units under control conditions and during BMI/phaclofen application. Each histogram shows accumulated responses to 10 whisker deflection trials at the indicated angle. $0^{\circ}$ represents an initially caudal deflection, and $90^{\circ}$ represents an initially dorsal deflection. Each tick mark represents $1 \mathrm{spike} / 1 \mathrm{msec}$ bin. Stimulus waveforms are shown below and are 500 msec in total duration; plateau duration is $200 \mathrm{msec}$.

Consistent with the increase in the proportion of tonic cells, the average plateau activity increased more so than the spontaneous activity (Fig. $2 A, B$ ). The persistence of elevated plateau activity over an extended $(400 \mathrm{msec})$ plateau period illustrates that activity during the $200 \mathrm{msec}$ plateau represents true sustained or steady-state activity (Fig. $2 C$ ). We compared increases in spontaneous and plateau activities in terms of both absolute firing rates and relative fold-changes (response with drug divided by control response). By both measures the greater increase in plateau activity was significant (one-tail paired $t$ tests, all directions: absolute $p<0.001$; relative $p=0.04$ ). The directional properties of plateau activity were not affected by $\mathrm{BMI} /$ phaclofen, as assessed by both the directional tuning ratio and tuning category measures (paired $t$ tests, $p>0.05$ ).

Transient responses to the onset and offset of whisker deflection were also substantially altered by disinhibition. The time of decay to half-maximal amplitude of the ON response in Figure $2 A$ increased from 2 to $35 \mathrm{msec}$, resulting in a much larger overall response magnitude (Table 1). Similar although less dramatic results were obtained for the OFF response. Surprisingly, however, neither the magnitude nor timing of the earliest components of response transients were affected, as illustrated in the highresolution histograms of Figure 3. For each cell we computed response magnitudes for different durations after response onset in both control and disinhibited conditions and corrected these measures by subtracting corresponding spontaneous activities. Only after the first $5 \mathrm{msec}$ of the $\mathrm{ON}$ response do spike counts in the two conditions become significantly different $(p<0.05$, one-tailed paired $t$ test); OFF responses were corrected by subtracting plateau activity and did not differ until after 9 msec. Tuning ratios for the $\mathrm{ON}$ response decreased significantly under blockade of inhibition (paired $t$ test, $p<0.01$ ), but the distribution of cells across statistically based tuning categories was not affected.

\section{DISCUSSION}

The main result of the present study is that antagonism of GABA receptors elevates tonic responsiveness in VPm neurons. We interpret the elevated tonic activity to be an expression, or unmasking, of tonic, stimulus-evoked excitatory inputs from brain-
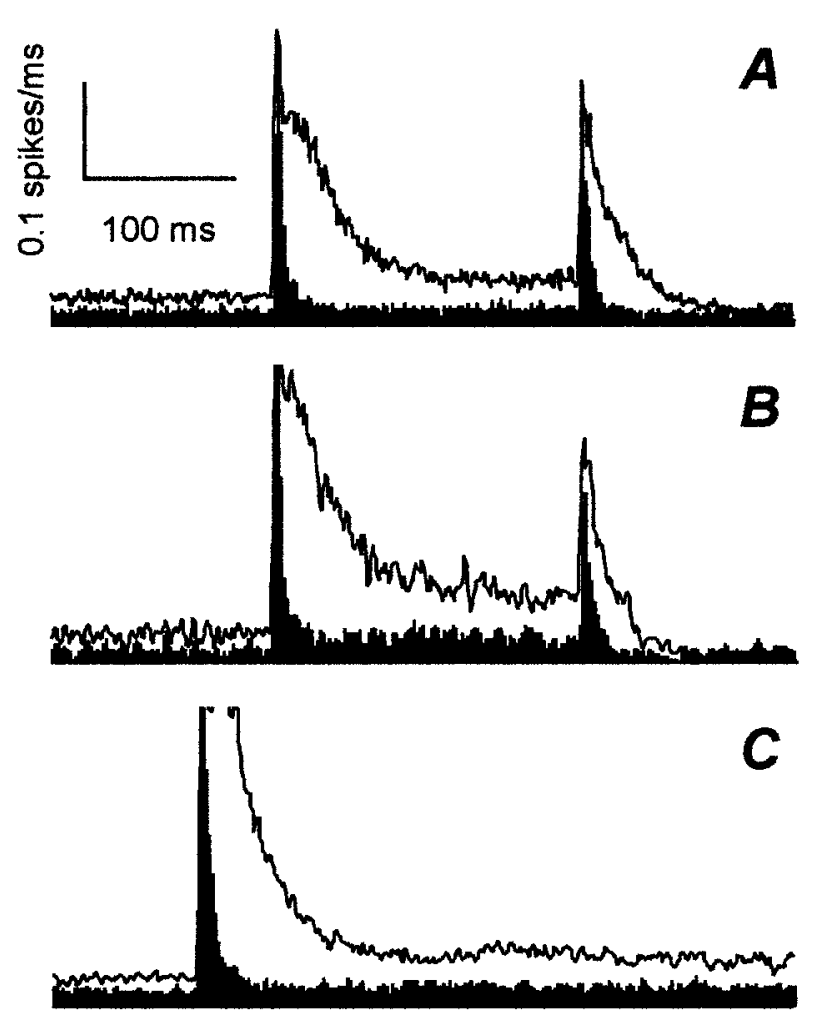

Figure 2. Effects of GABA receptor antagonism on population responses. Filled and open PSTHs show mean population activity recorded under control and experimental conditions, respectively. $A$, Responses accumulated for all deflection angles. $B$, Responses for angles evoking the greatest plateau activity for each cell. $C$, Responses of seven cells to deflections with a $400 \mathrm{msec}$ plateau, accumulated over all deflection angles. The ON response is plotted earlier in $C$ to illustrate the full duration of the $400 \mathrm{msec}$ plateau response. PSTHs in $B$ and $C$ are smoothed with a 3 msec moving boxcar average, and peak ON responses are truncated at 0.3 spikes $/ \mathrm{msec}$. 


\begin{tabular}{lccl}
\hline \multicolumn{3}{l}{ Table 1. Comparison of control and disinhibited activities } & \\
& Control & BMI + phaclofen & Fold-change \\
\hline $\mathrm{ON}^{a}$ & $2.9 \pm 1.4$ & $11.2 \pm 6.3$ & 4.03 \\
$\mathrm{OFF}^{a}$ & $2.1 \pm 1.1$ & $5.8 \pm 3.4$ & 2.88 \\
Spontaneous $^{b}$ & $15.6 \pm 7.6$ & $30.3 \pm 20.0$ & 2.21 \\
Plateau $^{b}$ & & & \\
$\quad$ All angles & $18.3 \pm 8.5$ & $52.6 \pm 36.0$ & 2.88 \\
$\quad$ Maximum angle & $30.3 \pm 12.7$ & $81.9 \pm 51.2$ & 2.72 \\
T-50\% & $11.8 \pm 8.3$ & $25.3 \pm 5.1$ & 2.42 \\
\end{tabular}

$\overline{\text { Values denote means } \pm \text { SD. Fold-change values were computed as averages of }}$ fold-changes calculated for each neuron, and therefore may differ slightly from values calculated from population means.

${ }^{a} \mathrm{ON}$ and $\mathrm{OFF}$ responses are reported in spikes per stimulus, computed for 75 and $50 \mathrm{msec}$ durations, respectively.

${ }^{b}$ Spontaneous and plateau activities are reported in spikes per second.

${ }^{c} \mathrm{~T}-50 \%$ is the time (milliseconds) of occurrence of the 50th percentile spike in the ON response ( $75 \mathrm{msec}$ window) of a neuron's PSTH accumulated over 80 trials.
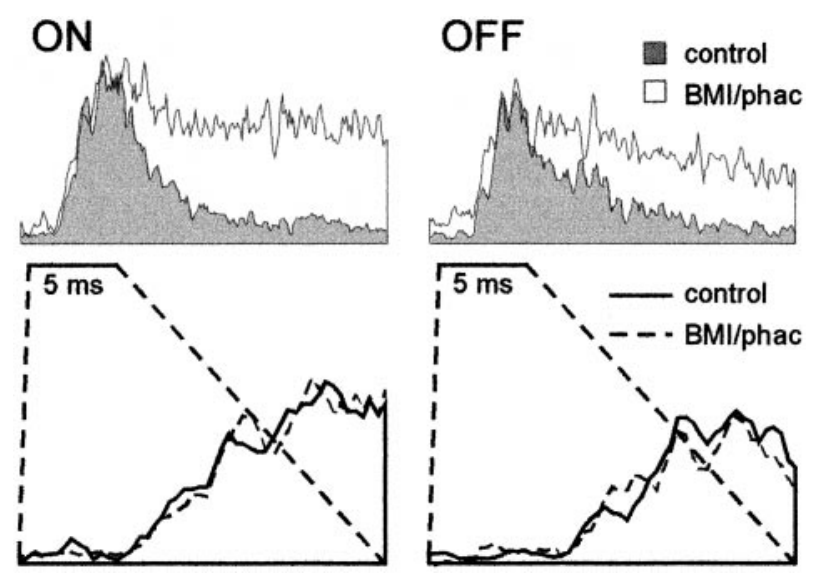

Figure 3. Effects of BMI/phaclofen on transient responses. Above and below are population PSTHs of ON and OFF responses accumulated over all deflection angles with $100 \mu \mathrm{sec}$ bins, smoothed with a $300 \mu \mathrm{sec}$ moving boxcar average. Above: filled histograms $=$ control ; open histograms $=$ BMI/phac. Five millisecond periods are shown below at higher resolution after BMI histograms were corrected by subtracting differences in spontaneous ( $O N$ response) and plateau (OFF response) activity. Below: solid lines $=$ control; dashed lines $=$ BMI/phac.

stem afferents that are not reflected in the output of VPm neurons under control conditions because of inhibitory suppression. This disinhibited tonic activity does not reflect a nonspecific increase in neuronal excitability, because plateau responses increased significantly more than spontaneous activity. Furthermore, even the proportional increases in plateau responses were significantly greater than those in spontaneous activity, which is opposite to the effect of BMI on cortical neurons (Kyriazi et al., 1996). Additionally, serving as a negative control, some cells were observed to increase their plateau activity only as much as spontaneous activity increased, and in other cells tonic responsiveness increased in a direction-selective fashion (Fig. 1 $A$ ). Taken together, the findings indicate that increased tonic responsiveness reflects an unmasking of specific excitatory inputs. We conclude that the increases in tonic activity during maintained deflections induced by BMI/phaclofen reveal specific stimulus-evoked influences of inhibition on the input-output relationship in VPm neurons, namely the filtering of slowly adapting activity.

Previous studies have also suggested that slowly adapting activity is suppressed at the thalamic level. Lee et al. (1994a,b) examined the effects of thalamic reticular nucleus lesions or direct bicuculline microiontophoresis on the "sustained" versus "tran- sient" nature of VPm responses to brief $(50 \mathrm{msec})$ whisker deflections. They observed a marked increase in the proportion of units classified as sustained. As suggested by our results (Fig. 2), however, an increase in activity over $50 \mathrm{msec}$ after stimulus may reflect a prolonged $\mathrm{ON}$ response, rather than sustained, steadystate activity per se. We chose a time period for computing plateau activity of $75-175 \mathrm{msec}$ after deflection onset to avoid this confound and confirmed with a $400 \mathrm{msec}$ stimulus that activity in this period truly reflects sustained responses. An important next step is to record from whisker-related "barrelette" neurons in brainstem nuclei under the present recording conditions to determine the extent of feedforward slowly adapting activity impinging on VPm neurons. Previous studies of these neurons reported that most are rapidly adapting (Shipley, 1974). Anesthesia, however, may alter important temporal aspects of neuronal responsiveness in the brainstem, as may have been the case for the negative findings reported in the thalamus by Vahle-Hinz and Hicks (1996).

In addition to unmasking tonic responses, antagonism of GABA receptors increased the duration, and hence magnitude, of the transient ON and OFF responses. The earliest 5-9 msec of these evoked responses, however, were not significantly affected. The delayed impact of inhibition on VPm ON and OFF responses is consistent with its origination in the Rt, which forms a feedback circuit with dorsal thalamic nuclei. The earliest whisker-evoked spikes arise in Rt $1.3 \mathrm{msec}$ after the first VPm responses (Hartings et al., 2000), and inhibitory postsynaptic currents in VPm elicited by Rt spikes have latencies of 1.5-3.1 msec (Cox et al., 1997), followed by $1.2 \mathrm{msec} 10-90 \%$ rise times (Huntsman and Huguenard, 2000). Thus several milliseconds are required for feedback Rt inhibition to impact thalamocortical signaling. These delays may establish a "relay" function in the thalamus for the early components of afferent signals. Interestingly, recordings from regular-spiking neurons in cortical layer IV demonstrate that the magnitude of their evoked responses is best predicted by the number of spikes occurring within these first 2-7 msec of VPm responses (Pinto et al., 2000). Furthermore, modeling of this circuit suggested that responses of regular-spiking barrel neurons are insensitive to thalamic spikes arriving after this "window of opportunity" (Pinto et al., 1996).

We hypothesize that the control of ongoing V Pm activity may indirectly regulate the responsiveness of cortical barrel neurons to transient thalamocortical signals. To illustrate this, we used the histograms in Figure $2 A$ as input to our population model of layer IV barrel circuitry (Pinto et al., 1996). The histogram accumulated under $\mathrm{BMI} /$ phaclofen iontophoresis produced a cortical ON response less than half the size of that elicited by the control histogram, despite the former's fourfold greater thalamic ON response (Table 1). The smaller cortical response is attributable to the higher ongoing (spontaneous) thalamic activity under $\mathrm{BMI} /$ phaclofen, which in the model, as in the real circuit (Brumberg et al., 1996), drives inhibitory barrel neurons more so than excitatory ones, narrowing the window of opportunity and thereby diminishing responses to transient thalamic inputs. When the 15 spikes/sec increase in spontaneous activity (Table 1) is subtracted from the BMI/phaclofen histogram, spontaneous firing of inhibitory barrel neurons returns to normal levels, and the cortical ON response recovers to $97 \%$ of its control value. The present experimental findings, together with these simulations, predict that acute lesions of Rt would reduce cortical responsiveness despite increases in spontaneous and stimulus-evoked thalamic activity.

\section{REFERENCES}

Alloway KD, Wallace MB, Johnson MJ (1994) Cross-correlation analysis of cuneothalamic interactions in the rat somatosensory system: influence of receptive field topography and comparisons with thalamocortical interactions. J Neurophysiol 72:1949-1972.

Brumberg JC, Pinto DJ, Simons DJ (1996) Spatial gradients and inhib- 
itory summation in the rat whisker barrel system. J Neurophysiol 76:130-140.

Cox CL, Huguenard JR, Prince DA (1997) Nucleus reticularis neurons mediate diverse inhibitory effects in thalamus. Proc Natl Acad Sci USA 94:8854-8859.

Dykes RW, Sur M, Merzenich MM, Kaas JH, Nelson RJ (1981) Regional segregation of neurons responding to quickly adapting, slowly adapting, deep and Pacinian receptors within thalamic ventroposterior lateral and ventroposterior inferior nuclei in the squirrel monkey (Saimiri sciuresu). Neuroscience 6:1687-1692.

Gottschaldt K-M, Vahle-Hinz C, Hicks TP (1983) Electrophysiological and micropharmacological studies on mechanisms of input-output transformation in single neurones of the somatosensory thalamus. In: Somatosensory integration in the thalamus (Macchi G, Rustioni A, Spreafico R, eds), pp 199-216. Amsterdam: Elsevier.

Harris RM, Hendrickson AE (1987) Local circuit neurons in the rat ventrobasal thalamus: a GABA immunocytochemical study. Neuroscience 21:229-236.

Hartings JA, Temereanca S, Simons DJ (2000) High responsiveness and direction sensitivity of neurons in the rat thalamic reticular nucleus to vibrissa deflections. J Neurophysiol 83:2791-2801.

Hicks TP, Metherate R, Landry P, Dykes RW (1986) Bicucullineinduced alterations of response properties in functionally identified ventroposterior thalamic neurones. Exp Brain Res 63:248-264.

Huntsman MM, Huguenard JR (2000) Nucleus-specific differences in $\mathrm{GABA}_{\mathrm{A}}$-receptor mediated inhibition are enhanced during thalamic development. J Neurophysiol 83:350-358.

Ito M (1988) Response properties and topography of vibrissa-sensitive VPM neurons in the rat. J Neurophysiol 60:1181-1197.

Kyriazi HT, Carvell GE, Brumberg JC, Simons DJ (1996) Quantitative effects of GABA and bicuculline methiodide on receptive field properties of neurons in real and simulated whisker barrels. J Neurophysiol 75:547-560.

Lee SM, Friedberg MH, Ebner FF (1994a) The role of GABA-mediated inhibition in the rat ventral posterior medial thalamus. I. Assessment of receptive field changes following thalamic reticular nucleus lesions. J Neurophysiol 71:1702-1715.

Lee SM, Friedberg MH, Ebner FF (1994b) The role of GABA-mediated inhibition in the rat ventral posterior medial thalamus. II. Differential effects of GABAa and GABAb receptor antagonists on responses of VPM neurons. J Neurophysiol 71:1716-1726.

Lichtenstein SH, Carvell GE, Simons DJ (1990) Responses of rat trigeminal ganglion neurons to movements of vibrissae in different directions. Somatosens Mot Res 7:47-65.

Pinault D, Bourassa J, Deschenes M (1995) The axonal arborization of single thalamic reticular neurons in the somatosensory thalamus of the rat. Eur J Neurosci 7:31-40.

Pinto DJ, Brumberg JC, Simons DJ, Ermentrout GB (1996) A quantitative population model of whisker barrels: re-examining the WilsonCowan equations. J Comput Neurosci 3:247-264.

Pinto DJ, Brumberg JC, Simons DJ (2000) Circuit dynamics and coding strategies in rodent somatosensory cortex. J Neurophysiol 83:1158-1166

Powell TPS, Mountcastle VB (1959) Some aspects of the functional organization of the cortex of the postcentral gyrus of the monkey: a correlation of findings obtained in a single unit analysis with cytoarchitecture. Johns Hopkins Hosp Bull 105:133-162.

Salt TE (1989) Gamma-aminobutyric acid and afferent inhibition in the cat and rat ventrobasal thalamus. Neuroscience 28:17-26.

Shipley MT (1974) Response characteristics of single units in the rat's trigeminal nuclei to vibrissa displacements. J Neurophysiol 37:73-90.

Simons DJ (1983) Multi-whisker stimulation and its effects on vibrissa units in rat SmI barrel cortex. Brain Res 276:178-182.

Simons DJ, Carvell GE (1989) Thalamocortical response transformation in the rat vibrissa/barrel system. J Neurophysiol 61:311-330.

Spacek J, Lieberman AR (1974) Ultrastructure and three-dimensional organization of synaptic glomeruli in the rat somatosensory thalamus. J Anat 117:487-516.

Sugitani M (1979) Electrophysiological and sensory properties of the thalamic reticular neurones related to somatic sensation in rats. J Physiol (Lond) 290:79-95.

Vahle-Hinz C, Hicks TP (1996) GABA-mediated inhibition shapes phasic responses in the rat's thalamic ventrobasal complex (VB). Soc Neurosci Abstr 1:97.

Waite PME (1973) The responses of cells in the rat thalamus to mechanical movements of the whiskers. J Physiol (Lond) 228:541-561.

Zucker E, Welker WI (1969) Coding of somatic sensory input by vibrissae neurons in the rat's trigeminal ganglion. Brain Res 12:138-156. 